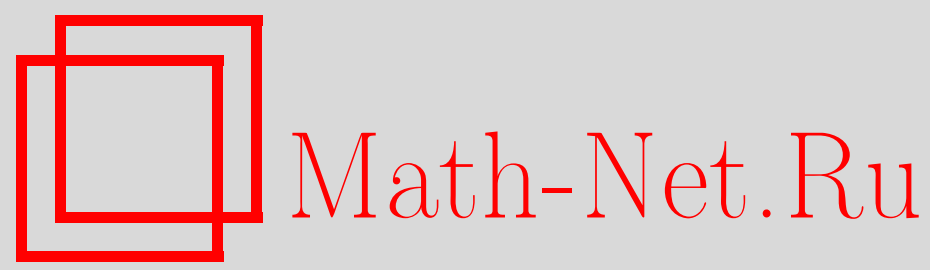

В. Ю. Протасов, Об убывании бесконечных произведений тригонометрических полиномов, Матем. заметки, 2002, том 72, выпуск 6, 892-908

DOI: https://doi.org/10.4213/mzm475

Использование Общероссийского математического портала Math-Net.Ru подразумевает, что вы прочитали и согласны с пользовательским соглашением http://www.mathnet.ru/rus/agreement

Параметры загрузки:

IP : 54.92 .164 .108

26 апреля 2023 г., $17: 08: 37$

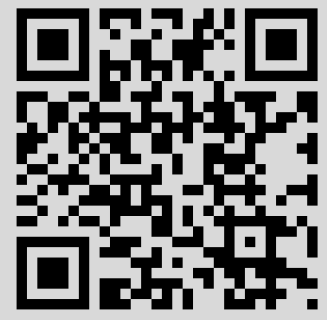




\section{ОБ УБЫВАНИИ БЕСКОНЕЧНЫХ ПРОИЗВЕДЕНИЙ ТРИГОНОМЕТРИЧЕСКИХ ПОЛИНОМОВ}

В. Ю. Протасов

В работе рассматриваются бесконечныепроизведения вида $f(\xi)=\prod_{k=1}^{\infty} m_{k}\left(2^{-k} \xi\right)$, где $\left\{m_{k}\right\}$ - произвольная последовательность тригонометрических полиномов степени не выше $n$, равномерно ограниченная по норме и такая, что $m_{k}(0)=1$ для всех $k$. Доказывается, что $f(\xi)$ не может убывать на бесконечности быстрее, чем $O\left(\xi^{-n}\right)$. Представлены условия на последовательность $\left\{m_{k}\right\}$, при которых максимальная скорость убывания достигается. Данный результат применим в теории нестационарных всплесков и нестационарных подразделительных схем. В частности, он ограничивает гладкость нестационарных всплесков длиной носителя порождающих функций. Это обобщает хорошо известные подобные результаты для стационарных последовательностей полиномов (когда все $m_{k}$ равны). В нескольких примерах мы показываем, что ослабление условия равномерной ограниченности по норме может привести к экспоненциальному убьванию.

Библиография: 21 названия.

1. Введение. В работе будут использованы следуюшие обозначения: $\mathbb{N}, \mathbb{R}, \mathbb{C}$ множества соответственно натуральных, действительньх и комплексных чисел, $\mathscr{P}_{n}-$ пространство алгебраических полиномов над $\mathbb{C}$ степени не вьше $n, \mathscr{L}_{1}$ - пространство суммируемых функций на $\mathbb{R}, S^{\prime}$ - пространство обобщенных функций медленного роста (двойственное к пространству Шварца $S$ ); для данных функций $g_{0}(x)$ и $g_{1}(x)$ обозначаем $g_{1}=o\left(g_{0}\right)$ при $x \rightarrow \infty$, если $g_{1} / g_{0} \rightarrow 0$ при $x \rightarrow \infty$; также $g_{1}=O\left(g_{0}\right)$ при $x \rightarrow \infty$, если существует такая константа $C>0$, что $\left|g_{1}\right| \leqslant C\left|g_{0}\right|$ для всех достаточно больших $x$. Мы будем иметь дело с полиномами вида

$$
m(\xi)=\sum_{s=0}^{n} c_{s} e^{-i s \xi}
$$

т.е. с тригонометрическими полиномами, не имеющими положительных степеней. Через $\widetilde{m}$ обозначается соответствуюший алгебраический полином

$$
\widetilde{m}(z)=\sum_{s=0}^{n} c_{s} z^{s}, \quad z \in \mathbb{C}
$$

Работа выполнена при финансовой поддержке Российского фонда фундаментальных исследований, гранты № 99-01-00357 и № 00-15-96109, а также фонда ИНТАС, грант № 99-01-080. 
таким образом, $m(\xi)=\widetilde{m}\left(e^{-i \xi}\right)$. Как обычно, мы обозначаем $\|m\|=\sup _{\xi \in \mathbb{R}}|m(\xi)|$. Для соответствующего алгебраического полинома через $\|\widetilde{m}\|$ обозначим норму на единичной окружности: $\|\widetilde{m}\|=\sup _{z \in \mathbb{C},|z|=1}|\widetilde{m}(z)|$. Таким образом, $\|m\|=\|\widetilde{m}\|$. Мы предполагаем всегда, что старший коэффициент $c_{n}$ ненулевой, т.е. $\operatorname{deg} m=\operatorname{deg} \widetilde{m}=n$. Далее, скорость убывания данной функции $g(\xi)$ (обозначается $\mathbf{d}(g)$ ) - это наибольшее целое число $l$ такое, что $g(\xi)=o\left(\xi^{-l}\right)$ при $|\xi| \rightarrow \infty$; если $g$ убывает быстреевсякой степени, то полагается $\mathbf{d}(g)=+\infty$, если же таких целых $l$ вовсе не существует, то $\mathbf{d}(g)=-\infty$.

Для произвольной последовательности полиномов $\left\{m_{k}\right\}_{k \in \mathbb{N}}$ такой, что $m_{k}(0)=1$, $k \in \mathbb{N}$, рассмотрим следующее бесконечное произведение:

$$
f(\xi)=\prod_{k=1}^{\infty} m_{k}\left(2^{-k} \xi\right) .
$$

Подобные произведения естественно возникают в теории всплесков (wavelets), при изучении подразделительных схем (subdivision schemes), используемых в теории приближений и в дизайне кривых и поверхностей (мы обсудим это позже), а также в некоторых проблемах теории вероятностей (см. [1]-[3]). Произведения такого типа используются также при анализе фрактальных кривых (например, кривых Де Рама, см. [4], [5]), сверток Бернулли (см. [6], [7]) и в некоторых задачах комбинаторной теории чисел (см. [8]).

При некоторых довольно общих условиях (например, когда нормы и степени всех полиномов равномерно ограничены) произведение (1) сходится равномерно на каждом компакте и, следовательно, представляет аналитическую функцию. Насколько быстро эта функция может убывать на бесконечности? В данной работе мы изучаем скорость убывания функции $f$ в терминах последовательности $\left\{m_{k}\right\}$.

В частном случае, когда все полиномы $m_{k}$ равны между собой, данная задача исследовалась чрезвычайно подробно (см. список литературы). Легко видеть, что в этом случае обратное преобразование Фурье функции $f_{m}(\xi)=\prod_{k=1}^{\infty} m\left(2^{-k} \xi\right)$ существует, по крайней мере, в смысле обобщенных функций и удовлетворяет масштабирующему уравнению:

$$
\check{f}_{m}(x)=2 \sum_{s=0}^{n} c_{s} \check{f}_{m}(2 x-s),
$$

где $c_{0}, \ldots, c_{n}-$ коэффициенты полинома $m$. Данное уравнение играет исключительную роль в теории всплесков (см., например, [9]), а также в теории приближений и дизайне кривьх (см. [4], [5], [10], [11]). Нетрудно показать, что либо уравнение (2) вообще не имеет $\mathscr{L}_{1}$-решений, либо функция $\check{f}_{m}$ является его единственньм решением (в том случае, если $\left.\check{f}_{m} \in \mathscr{L}_{1}\right)$. Если скорость убывания функции $f_{m}$ больше чем 1 , то $\check{f}_{m}$ действительно принадлежит $\mathscr{L}_{1}$ и, более того, по крайней мере $\mathbf{d}\left(f_{m}\right)$ два раза дифференцируема. Поэтому гладкость решений масштабирующих уравнений может быть оценена с помощью скорости убьвания соответствуюших полиномиальных произведений $f_{m}$. Такая идея успешно использовалась в [12]-[14] и многих других работах по всплескам и масштабирующим функциям. Хорошо известно, что скорость убывания функции $f_{m}(\xi)$ не может превосходить $n-1$, где $n=\operatorname{deg} m$, и эта максимальная скорость достигается только для полинома

$$
m(\xi)=\left(\frac{e^{-i \xi}+1}{2}\right)^{n}
$$


который мы обозначим через $w_{n-1}(\xi)$. Соответствующее произведение

$$
f_{w}(\xi)=\prod_{k=1}^{\infty} w_{n-1}\left(2^{-k} \xi\right)
$$

является преобразованием Фурье кардинального В-сплайна порядка $n-1: B_{n-1}(x)=$ $\chi_{[0,1]}(x) * \cdots * \chi_{[0,1]}(x)$ (здесь $n-1$ сверток, $\chi_{[0,1]}(x)-$ характеристическая функция отрезка $[0,1])$. В самом деле,

$$
f_{w}(\xi)=\prod_{k=1}^{\infty} \frac{e^{-i 2^{-k} \xi}+1}{2}=\frac{\left[i\left(e^{-i \xi}-1\right)\right]^{n}}{\xi^{n}}=\widehat{B}_{n-1}(\xi)
$$

Итак, для любого полинома $m$ степени $n$ имеем $\mathbf{d}\left(f_{m}\right) \leqslant n-1$. Это неравенство обращается в равенство только в случае $w_{n-1}$, для которого, более того, $f_{w}(\xi)=O\left(\xi^{-n}\right)$. Это так называемая максимальность В-сплайна (см. [4], [15], где приведены соответствующие доказательства; см. также [16], [17] о свойствах и применениях В-сплайнов). Данное свойство означает, в частности, что гладкость всплесков с носителем длины $n$ не превосходит $n-1$.

Возникает вопрос: насколько быстро может убьвать функция (1) в общем случае, когда полиномы $m_{k}, k \in \mathbb{N}$, различны и выбираются произвольно? Можно ли получить более быстрый рост, выбирая подходящую последовательность полиномов степени не выше $n$ ? Этот вопрос появился при изучении нестационарных всплесков с компактным носителем, представленных впервые в работах [18], [19]. Такиевсплески такжестроятся с помощью произведения (1), однако полиномы $\left\{m_{k}\right\}$ в этом случае уже не обязаны совпадать. Задача была сформулирована профессором И. Я. Новиковьп в 1999 году: может ли произведение (1), при условии, что степени полиномов $m_{k}$ не превосходят $n$, а сами полиномы равномерно ограничены по норме, иметь бесконечную скорость убьвания, т.е. убывать быстрее, чем полиномиально? Положительный ответ на этот вопрос привел бы к построению бесконечно гладких нестационарных всплесков с равномерно ограниченными носителями. Более точно: было бы возможно получить систему нестационарных всплесков, у которых порождающие функции всех уровней $\left\{F_{0 j}(t)\right\}_{j \geqslant 0}$ являются бесконечно гладкими и при этом носители функций $F_{0 j}\left(2^{-j} t\right), j \geqslant 0$, содержатся в некотором отрезке $[-C, C]$ (см. [20]). Мы доказываем теорему 1 , согласно которой ответ на данньй вопрос отрищательный. Оказьвается, что скорость убывания не только не может быть бесконечной, но и по-прежнему не может превосходить $n-1$. Это означает, в частности, что гладкость нестационарных всплесков не может превосходить максимальной из длин носителей функций $F_{0 j}\left(2^{-j} t\right), j \geqslant 0$. Таким образом, в нестационарном случае ситуация подобна той, которую мы имеем для стационарных всплесков.

Данный результат также ограничивает гладкость функций, порождаемых нестационарными подразделительными схемами (см. [21], где приведены описание и применения таких схем). Здесь ситуация оказывается полностью аналогичной стационарному случаю: если суммы абсолютных величин коэффициентов схемы на всех шагах равномерно ограничены, то показатель гладкости схемы не превосходит $n$. Оптимальной, как и в стационарном случае, оказьвается схема, соответствующая кардинальному В-сплайну. 
Таким образом, максимальность В-сплайнов распространяется на широкий класс полиномиальных произведений: на произведения вида (1) для всех возможных последовательностей полиномов $\left\{m_{k}\right\}$ (с равномерно ограниченньми степенями и нормами). Более того, мы покажем, что если полиномиальное произведение имеет максимальную скорость убывания $n-1$, то соответствующая последовательность $m_{k}$ сходится к полиному $w_{n-1}$, т.е. $\left\|m_{k}-w_{n-1}\right\| \rightarrow 0$ при $k \rightarrow \infty$. В нескольких примерах мы покажем, что ни одно из условий теоремы 1 не может быть ослаблено. В частности (пример 2), если отказаться от равномерной ограниченности полиномов по норме, то можно построить произведение $f$, убывающее экспоненцильно. Далее, в теореме 2, устанавливаются условия на последовательность $\left\{m_{k}\right\}$, при которых произведение $f$ имеет быстрейшее возможное убывание, т.е. убывает как $O\left(\xi^{-n}\right)$.

\section{2. Основной результат.}

ТЕОремА 1. Предположим, что нам дана последовательность тригонометрических полиномов $\left\{m_{k}\right\}_{k \in \mathbb{N}}$ и числа $n \in \mathbb{N}, M \geqslant 1$ mакие, что $m_{k}(0)=1$, $\operatorname{deg} m_{k} \leqslant n,\left\|m_{k}(\cdot)\right\| \leqslant M$ для всех $k \in \mathbb{N}$. Тогда скорость убывания функиии

$$
f(\xi)=\prod_{k=1}^{\infty} m_{k}\left(2^{-k} \xi\right)
$$

не превосходит $n-1$. Более того, если скорость убывания равна $n-1$, то $m_{k} \rightarrow w_{n-1}$ npu $k \rightarrow \infty$.

ДокАЗАТЕЛЬСТво. Предположим, что скорость убьвания не меньше, чем $n-1$. Это означает, что

$$
\xi^{n-1} f(\xi) \rightarrow 0 \quad \text { при }|\xi| \rightarrow \infty .
$$

Положим $\sigma=1 /(2 n M)$. Для каждого $\xi \in[-\sigma, \sigma], j \geqslant 0$ и $N \in \mathbb{N} \cup\{+\infty\}$ имеем

$$
\left|\prod_{k=1}^{N} m_{j+k}\left(2^{-k} \xi\right)\right|>\frac{1}{2} \text {. }
$$

Действительно, в силу неравенства Бернштейна $\left\|m_{j+k}^{\prime}\right\| \leqslant n M$, поэтому

$$
\begin{aligned}
\left|m_{j+k}\left(2^{-k} \xi\right)\right| & \geqslant\left|m_{j+k}(0)\right|-2^{-k}|\xi| \cdot \| m_{j+k}^{\prime}\left|\geqslant 1-n M 2^{-k}\right| \xi \mid \\
& \geqslant 1-n M 2^{-k} \sigma=1-2^{-k-1} .
\end{aligned}
$$

Следовательно,

$$
\left|\prod_{k=1}^{N} m_{j+k}\left(2^{-k} \xi\right)\right| \geqslant \prod_{k=1}^{N}\left(1-2^{-k-1}\right)>\frac{1}{2} .
$$

Обозначим через $p$ наименьшее целое число, для которого $2^{p} \geqslant 8 \pi n M$. Теперь для произвольных $\delta \in(0, \sigma)$ и $l \in \mathbb{N}$ получаем

$$
\begin{aligned}
f\left(2 \pi \cdot 2^{l}+\delta\right)= & \prod_{k=1}^{l} m_{k}\left(2 \pi \cdot 2^{l-k}+2^{-k} \delta\right) \prod_{k=1}^{p} m_{l+k}\left(2 \pi \cdot 2^{-k}+2^{-l-k} \delta\right) \\
& \times \prod_{k=1}^{\infty} m_{l+p+k}\left(2 \pi \cdot 2^{-p-k}+2^{-l-p-k} \delta\right) .
\end{aligned}
$$


Применив (4) для $\xi=\delta, j=0$ и $N=l$, получим

$$
\left|\prod_{k=1}^{l} m_{k}\left(2 \pi \cdot 2^{l-k}+2^{-k} \delta\right)\right|=\left|\prod_{k=1}^{l} m_{k}\left(2^{-k} \delta\right)\right|>\frac{1}{2}
$$

теперь применим (4) для $\xi=2 \pi \cdot 2^{-p}+2^{-l-p} \delta, j=l+p$ и $N=+\infty$, получив

$$
\left|\prod_{k=1}^{\infty} m_{l+p+k}\left(2 \pi \cdot 2^{-p-k}+2^{-l-p-k} \delta\right)\right|>\frac{1}{2}
$$

Таким образом,

$$
\frac{\left|f\left(2 \pi \cdot 2^{l}+\delta\right)\right|}{4} \geqslant\left|m_{l+1}\left(\pi+2^{-l-1} \delta\right) m_{l+2}\left(\frac{\pi}{2}+2^{-l-2} \delta\right) \cdots m_{l+p}\left(2^{1-p} \pi+2^{-l-p} \delta\right)\right| .
$$

По предположению $2^{l(n-1)} f\left(2 \pi \cdot 2^{l}+\delta\right) \rightarrow 0$ при $l \rightarrow \infty$, следовательно,

$$
\begin{gathered}
2^{l(n-1)} m_{l+1}\left(\pi+2^{-l-1} \delta\right) m_{l+2}\left(\frac{\pi}{2}+2^{-l-2} \delta\right) \cdots m_{l+p}\left(2^{1-p} \pi+2^{-l-p} \delta\right) \rightarrow 0 \\
\text { при } l \rightarrow \infty .
\end{gathered}
$$

Последнее вьполнено для всех $\delta \in(0, \sigma)$. Теперь зададим положительное $R$ и окружим каждую точку $\left\{e^{-2^{1-k} \pi i}\right\}_{k=1}^{p}$ и $\left\{e^{-3 \cdot 2^{1-k} \pi i}\right\}_{k=1}^{p}$ замкнутым кругом радиуса $R$ (с центром в этой точке). Обозначим эти круги через $\left\{\beta_{k}\right\}_{k=1}^{p}$ и $\left\{\gamma_{k}\right\}_{k=1}^{p}$ соответственно. Таким образом, мы получаем семейство из $2 p$ одинаковых кругов, в котором два круга $\beta_{1}$ и $\gamma_{1}$ совпадают. Сделаем радиус $R$ настолько малым, чтобы все остальные круги не пересекались.

Возьмем произвольное $l \geqslant 0$ и рассмотрим цепочку соответствующих алгебраических полиномов $\widetilde{m}_{l+1}, \ldots, \widetilde{m}_{l+p}$. Пусть круг $\beta_{k}$ содержит $s_{k}$ корней полинома $\widetilde{m}_{l+k}(\mathrm{c}$ учетом кратности корней). Если $s_{k}>0$, то обозначим эти корни через $z_{1}^{(k)}, \ldots, z_{s_{k}}^{(k)}$, а все остальные корни $\widetilde{m}_{l+k}$ через $z_{s_{k}+1}^{(k)}, \ldots, z_{n_{k}}^{(k)}$, где $n_{k}=\operatorname{deg} \widetilde{m}_{l+k}$. Имеем

$$
\widetilde{m}_{l+k}(z)=A_{k} \tilde{\psi}_{k}(z) \tilde{q}_{k}(z)
$$

где

$$
\tilde{\psi}_{k}(z)=\prod_{j=1}^{s_{k}}\left(z-z_{j}^{(k)}\right), \quad \tilde{q}_{k}(z)=\prod_{\nu=s_{k}+1}^{n_{k}}\left(\frac{z-b_{k}}{z_{\nu}^{(k)}-b_{k}}-1\right)
$$

$b_{k}=e^{-2^{1-k} \pi i}-$ центр круга $\beta_{k}$ и $A_{k}$ - некоторая константа (в случае $s_{k}=0$ полагаем $\tilde{\psi}_{k}=1$, если же $s_{k}=n_{k}$, то $\tilde{q}_{k}=1$ ). Конечно же, величины $s_{k}, A_{k}, \tilde{\psi}_{k}, \tilde{q}_{k}$ зависят не только от $k$, но и от $l$, но для краткости обозначений мы не будем записьвать это. Для любой точки $z$ на единичной окружности имеем $\left|z-z_{j}\right| \leqslant 1+R$ для $j=1, \ldots, s_{k}$ и

$$
\left|\frac{z-b_{k}}{z_{\nu}^{(k)}-b_{k}}-1\right|<\frac{2}{R}+1 \quad \text { для } \quad \nu=s_{k}+1, \ldots, n_{k} .
$$


Следовательно, $\left\|\tilde{\psi}_{k}\right\| \leqslant(1+R)^{s_{k}}$ и $\left\|\tilde{q}_{k}\right\| \leqslant(2 / R+1)^{n_{k}-s_{k}}$. Теперь, применив неравенство $\left\|\widetilde{m}_{l+k}\right\| \geqslant\left|\widetilde{m}_{l+k}(1)\right|=1$, получим

$$
\begin{aligned}
& \left|A_{k}\right| \geqslant \frac{\left\|\tilde{m}_{l+k}\right\|}{\left\|\tilde{\psi}_{k}\right\| \cdot\left\|\tilde{q}_{k}\right\|} \geqslant \frac{1}{(1+R)^{s_{k}}(2 / R+1)^{n_{k}-s_{k}}} \\
& \geqslant \frac{1}{(1+R)^{n}(2 / R+1)^{n}}=\left(\frac{2}{R}+R+3\right)^{-n} \text {. }
\end{aligned}
$$

Наконец, обозначим через $\bar{\beta}_{k}$ замкнутый круг радиуса $R / 2$ с центром в точке $b_{k}$. Для каждого $z \in \bar{\beta}_{k}, \nu=s_{k}+1, \ldots, n_{k}$, имеем

$$
\left|\frac{z-b_{k}}{z_{\nu}^{(k)}-b_{k}}\right|<\frac{1}{2}
$$

поэтому $\left|\tilde{q}_{k}(z)\right|>2^{-n}$. Совместив это с (7), получаем

$$
\left|\widetilde{m}_{l+k}(z)\right| \geqslant 2^{-n}\left(\frac{2}{R}+R+3\right)^{-n}\left|\tilde{\psi}_{k}(z)\right| \quad \text { для каждого } z \in \bar{\beta}_{k} .
$$

Из этого следует, что для достаточно больших $l$, а именно для таких, что $2^{-l} \sigma \leqslant R$, имеем

$$
\left|\prod_{k=1}^{p} m_{l+k}\left(2^{1-k} \pi+2^{-l-k} \delta\right)\right| \geqslant\left[2^{-n}\left(\frac{2}{R}+R+3\right)^{-n}\right]^{p}\left|\prod_{k=1}^{p} \psi_{k}\left(2^{1-k} \pi+2^{-l-k} \delta\right)\right|
$$

где $\psi_{k}$ - тригонометрический полином, соответствующий полиному $\tilde{\psi}$, т.е. $\psi(\xi)=$ $\tilde{\psi}\left(e^{-i \xi}\right)$. Подставив это в $(6)$, получим

$$
2^{l(n-1)} \prod_{k=1}^{p} \psi_{k}\left(2^{1-k} \pi+2^{-l-k} \delta\right) \rightarrow 0 \quad \text { при } \quad l \rightarrow \infty
$$

Мы приходим к заключительному шагу в доказательстве. Окружим каждьй корень полинома $\prod_{k=1}^{p} \tilde{\psi}_{k}(z)$ кругом радиуса $r=\sigma /\left(2^{l+p} \pi S\right)$, где $S=\sum_{k=1}^{p} s_{k}$. Каждый из этих кругов отсекает на единичной окружности дугу длиной меньшей $\pi r$. Следовательно, общая длина множества

$$
\Delta_{1}=\left\{\delta \in(0, \sigma): \exists j \leqslant s_{1}\left|e^{-i\left(\pi+\delta 2^{-l-1}\right)}-z_{j}^{(1)}\right|<r\right\}
$$

меньше, чем $2^{l+1} \pi r s_{1}$. Точно так же показываем, что при любом $k=2, \ldots, p$ общая длина множества

$$
\Delta_{k}=\left\{\delta \in(0, \sigma): \exists j \leqslant s_{k}\left|e^{-i\left(\pi 2^{-k}+\delta 2^{-l-k}\right)}-z_{j}^{(k)}\right|<r\right\}
$$


меньше, чем $2^{l+k} \pi r s_{k}$. Следовательно, общая длина всех множеств $\Delta_{1}, \ldots, \Delta_{p}$ меньше, чем

$$
2^{l} \pi r \sum_{k=1}^{p} 2^{k} s_{k} \leqslant 2^{l} \pi r 2^{p} \sum_{k=1}^{p} s_{k}=2^{l+p} \pi r S=\sigma .
$$

Следовательно, существует точка $\delta_{0} \in(0, \sigma)$, не принадлежащая ни одному из этих множеств. Для этой точки имеем

$$
\left|\prod_{k=1}^{p} \psi_{k}\left(2^{1-k} \pi+2^{1-l-k} \delta_{0}\right)\right| \geqslant r^{S}
$$

Поскольку $S$ не превосходит общего числа корней полиномов $\left\{m_{l+k}\right\}_{k=1}^{p}$, то $S \leqslant n p$; следовательно,

$$
\begin{aligned}
r^{S} & =\left(\frac{\sigma}{2^{l+p} \pi S}\right)^{S}=\left(2^{l+p+1} M n \pi S\right)^{-S} \geqslant 2^{-S(l+p+1)}(M n \pi n p)^{-n p} \\
& \geqslant 2^{-S l}\left(2^{p+1} M n^{2} \pi p\right)^{-n p}
\end{aligned}
$$

Итак,

$$
\left|\prod_{k=1}^{p} \psi_{k}\left(2^{1-k} \pi+\delta_{0} 2^{-l-k}\right)\right| \geqslant 2^{-S l}\left(2^{p+1} M n^{2} \pi p\right)^{-n p} .
$$

Теперь, применив $(8)$, имеем $2^{l(n-1)} \cdot 2^{-l S} \rightarrow 0$ при $l \rightarrow+\infty$, поэтому $S \geqslant n$.

Напомним, что мы подсчитали количество всех корней полинома $\widetilde{m}_{l+k}$ в круге $\beta_{k}$ и вычислили сумму $S$ этих чисел для $k$ от 1 до $p$. Мы показали, что для каждого $l \geqslant 0$ эта сумма не меньше $n$. Точно так же доказьвается, что аналогичная сумма, посчитанная для кругов $\left\{\gamma_{k}\right\}_{k=1}^{p}$ также больше или равна $n$. Теперь совместим эти два факта: рассмотрим общее число корней полиномов $\widetilde{m}_{l+k}$ в кругах $\beta_{k}$ и $\gamma_{k}$ и вычислим сумму $S_{l}$ таких чисел для всех $k$ от 1 до $p$. Как мы показали, для каждого $l \geqslant 0$ возможны только два случая:

1) $S_{l}=n$, в этом случае все соответствующие корни лежат в круге $\beta_{0}$;

2) $S_{l} \geqslant n+1$.

Докажем, что для достаточно больших $l$ имеет место случай 1$)$. Для этого возьмем целое $N$ и оценим общее число корней полиномов $\widetilde{m}_{l+1}, \ldots, \widetilde{m}_{l+p+N}$. Очевидно, всего корней не более $n(p+N)$. С другой стороны, число корней не меньше $\sum_{j=0}^{N} S_{j}$, поскольку каждьй корень в этой сумме считается только один раз. Предположим теперь, что для $M$ цепочек полиномов $\left\{\tilde{m}_{l+j+k}\right\}_{k=1}^{p}$ имеет место случай 2$)$. Тогда $\sum_{j=0}^{N} S_{l} \geqslant$ $n(N+1)+M$, поэтому $n(p+N) \geqslant n(N+1)+M$. Таким образом, $M \leqslant n(p-1)$. Следовательно, начиная с некоторого номера $l_{0}$ для всех цепочек имеет место случай 1$)$. Таким образом, для каждого $k \geqslant l_{0}$ полином $\widetilde{m}_{k}$ имеет ровно $n$ корней в круге $\beta_{0}$. По условиям теоремы это означает, что полином $\operatorname{deg} \widetilde{m}_{k}$ имеет степень $n$ и все его $n$ корней лежат в круге $\beta_{0}$. Итак, при каждом $R>0$ существует номер $l_{0}(R)$ такой, что при любом $k \geqslant l_{0}(R)$ полином $\widetilde{m}_{k}$ имеет степень $n$ и все $n$ его корней лежат в круге $|z+1| \leqslant R$. Таким образом, все $n$ корней полинома $\tilde{m}_{k}$ стремятся к точке -1 при $k \rightarrow \infty$. Но поскольку для всех $k$ вьполнено $\widetilde{m}_{k}(1)=1$, из этого следует, что $\widetilde{m}_{k} \rightarrow((1+z) / 2)^{n}$ и, соответственно, $m_{k} \rightarrow w_{n-1}$ при $k \rightarrow \infty$. 
Если теперь предположить, что скорость убьвания функции $f(\xi)$ не меньше $n$, то, применив те же рассуждения, устанавливаем, что $m_{k} \rightarrow w_{n}$ при $k \rightarrow \infty$. Следовательно, $\operatorname{deg} m_{k} \geqslant n+1$ для всех достаточно больших $k$. Полученное противоречие завершает доказательство.

\section{3. Примеры и замечания.}

ЗАмЕчАнИЕ 1. Фактически, мы доказали более сильное утверждение: если скорость убывания функции $f$ с одной стороны неменьше $n-1$, т.е. $f(\xi)=o\left(\xi^{1-n}\right)$ при $\xi \rightarrow+\infty$ или $\xi \rightarrow-\infty$, то $m_{k} \rightarrow w_{n-1}$.

ЗАмЕчАниЕ 2 . Если степени полиномов $m_{k}$ не ограничены, то, вообще говоря, скорость убывания функции $f$ может быть бесконечна. Существуют последовательности полиномов, степени которых растут сколь угодно медленно, а функция $f$ убывает быстрее любой степени. Более точно сформулируем так.

УТВЕРЖДЕНИЕ 1. Для произвольной неубывающей последовательности натуральных чисел $\left\{a_{k}\right\}_{k \in \mathbb{N}}$ такой, что $\lim _{k \rightarrow \infty} a_{k}=+\infty$, существует последовательность тригонометрических полиномов $\left\{m_{k}\right\}_{k \in \mathbb{N}}$, для которой при всех $k$ $\left\|m_{k}\right\| \leqslant 1, m_{k}(0)=1, \operatorname{deg} m_{k} \leqslant a_{k}$ и при любом $d \geqslant 0 \quad f(\xi)=o\left(\xi^{-d}\right)$ nрu $|\xi| \rightarrow \infty$.

ДокАЗАТЕЛЬСТВо. Возьмем последовательность $d_{k}=\min \left\{a_{k}, k\right\}$ иполиномы $m_{k}=$ $w_{d_{k}-1}$. Легко видеть, что произведение

$$
f(\xi)=\prod_{k=1}^{\infty} m_{k}\left(2^{-k} \xi\right)
$$

сходится равномерно на каждом компакте в $\mathbb{R}$, т.е. функция $f$ корректно определена. Поскольку в этом случае $\left|m_{k}(\xi)\right| \leqslant 1$ при всех $k$ и $\xi$, вьполнено

$$
\begin{aligned}
|f(\xi)| & \leqslant\left|\prod_{k=r}^{\infty} m_{k}\left(2^{-k} \xi\right)\right|=\left|\prod_{k=r}^{\infty}\left(\frac{1+e^{-2^{-k} i \xi}}{2}\right)^{d_{k}}\right| \leqslant\left|\prod_{k=r}^{\infty}\left(\frac{1+e^{-2^{-k} i \xi}}{2}\right)\right|^{d_{r}} \\
& =\left|\widehat{B_{d_{r}-1}}\left(2^{1-r} \xi\right)\right| \leqslant 2^{r d_{r}}|\xi|^{-d_{r}} .
\end{aligned}
$$

Так как $d_{r} \rightarrow+\infty$ при $r \rightarrow \infty$, то функция $f$ убьвает быстрее любой степени $1 / \xi$. Другой пример таких последовательностей полиномов содержится в [20].

ЗАмЕчАниЕ 3. Согласно теореме 1 для любой ограниченной по норме последовательности тригонометрических полиномов степени $n$ функция $f$ неможет убывать быстрее, чем $O\left(\xi^{-n}\right)$. Эта максимальная скорость убывания достигается для стационарной последовательности $m_{k}=w_{n-1}, k \in \mathbb{N}$, но не только для нее. Например, для произвольного $a>0$ последовательность

$$
m_{k}(\xi)=\left(\frac{e^{-i \xi}+a^{(1 / 2)^{k}}}{1+a^{(1 / 2)^{k}}}\right)^{n}
$$

обладает тем же свойством быстрейшего убьвания. В теореме 2 мы установим критерий максимального убывания для последовательностей полиномов. 
ЗАмЕчаниЕ 4. Возникает естественный вопрос, насколько существенно в формулировке теоремы 1 условие равномерной ограниченности полиномов по норме? Нельзя ли заменить его более слабым условием равномерной сходимости произведения

$$
f(\xi)=\prod_{k=1}^{\infty} m_{k}\left(2^{-k} \xi\right)
$$

на каждом компакте? Ответ отрищательньй. Это может показаться парадоксальным. Если для ограниченной последовательности произведение $f(\xi)$ не может убывать слишком быстро, то для неограниченной последовательности оно должно быть еще больше и убывать еще медленнее. Тем не менее, в примере 2 мы увидим, что существует последовательность $\left\{m_{k}\right\}$, для которой $\operatorname{deg} m_{k}=2, m_{k}(0)=1$ при всех $k \geqslant 1$, и произведение $f(\xi)$ убывает экспоненциально при $|\xi| \rightarrow \infty$.

ЗАмечаниЕ 5. Для алгебраических полиномов аналог теоремы 1 также верен, хотя в этом случае он не так интересен и устанавливается гораздо проше. Нетрудно показать, что для произвольной последовательности алгебраических полиномов $\left\{p_{k}\right\}$ с равномерно ограниченными степенями и нормами (на единичной окружности) и со свойством $p_{k}(0)=1, k \in \mathbb{N}$, соответствующее произведение

$$
f(x)=\prod_{k=1}^{\infty} p_{k}\left(2^{-k} x\right)
$$

вообще не может стремиться к нулю при $x \rightarrow \infty$. Однако опять, как и в случае тригонометрических полиномов, без предположения равномерной ограниченности норм этот аналог теоремы 1 не верен (см. пример 1).

ПРИМЕР 1. Для произвольного четного числа $d \geqslant 2$ и для каждого $\varepsilon>0$ существует последовательность алгебраических полиномов $\left\{p_{k}\right\}$ такая, что для всех $k \geqslant 1$ $\operatorname{deg} p_{k} \leqslant d, p_{k}(0)=1$, произведение $f(x)=\prod_{k=1}^{\infty} p_{k}\left(2^{-k} x\right)$ сходится равномерно на каждом компакте и при этом

$$
|f(x)| \leqslant C e^{-|x|^{d-\varepsilon}}, \quad x \in \mathbb{R} .
$$

ДокАЗАТЕЛЬСТво. Для произвольного $\alpha \in(1,2)$ рассмотрим семейство полиномов первой степени

$$
Q_{k}(x)=1-\frac{2^{k}}{k^{\alpha}} x, \quad k \in \mathbb{N} .
$$

Произведение

$$
f_{Q}(x)=\prod_{k=1}^{\infty} Q_{k}\left(2^{-k} x\right)=\prod_{k=1}^{\infty}\left(1-\frac{x}{k^{\alpha}}\right)
$$

сходится равномерно на каждом компакте. Положим $x=y^{\alpha}$ для некоторого $y>2$ и обозначим через $I_{y}$ множество из четырех целых чисел $[y]-1,[y],[y]+1,[y]+2$, 
где символом $[y]$ обозначено наибольшее целое, не превосходящее $y$. Обозначим также $J_{y}=\mathbb{N} \backslash I_{y}$. Имеем

$$
f_{Q}(x)=\prod_{k \in J_{y}} Q_{k}\left(2^{-k} x\right) \prod_{k \in I_{y}} Q_{k}\left(2^{-k} x\right)
$$

и, кроме того, $Q_{k}\left(2^{-k} x\right) \neq 0$ для всех $k \in J_{y}$. Далее,

$$
\ln \left|\prod_{k \in J_{y}} Q_{k}\left(2^{-k} x\right)\right|=\sum_{k \in J_{y}} \ln \left|1-\left(\frac{y}{k}\right)^{\alpha}\right|=y \sum_{k \in J_{y}} \frac{1}{y} \ln \left|1-\left(\frac{k}{y}\right)^{-\alpha}\right| .
$$

Заметим, что

$$
\lim _{y \rightarrow+\infty} \sum_{k \in J_{y}} \frac{1}{y} \ln \left|1-\left(\frac{k}{y}\right)^{-\alpha}\right|=\int_{0}^{+\infty} \ln \left|1-t^{-\alpha}\right| d t
$$

(аккуратное доказательство предельного перехода предоставляем читателю). Если мы теперь обозначим

$$
b(\alpha)=\int_{0}^{+\infty} \ln \left|1-t^{-\alpha}\right| d t
$$

то получим

$$
\left|f_{Q}\left(y^{\alpha}\right)\right|=e^{b(\alpha) y+\omega(y)} \prod_{k \in I_{y}}\left|Q_{k}\left(2^{-k} y^{\alpha}\right)\right|
$$

где $\omega(y)=o(y)$ при $y \rightarrow+\infty$. Легко показать, что для достаточно больших $y$ произведение

$$
\prod_{k \in I_{y}}\left|Q_{k}\left(2^{-k} y^{\alpha}\right)\right|=\prod_{k \in I_{y}}\left|1-\left(\frac{k}{y}\right)^{-\alpha}\right|
$$

меньше единицы, поэтому

$$
\left|f_{Q}\left(y^{\alpha}\right)\right| \leqslant e^{b(\alpha) y+\omega(y)} .
$$

Заметим теперь, что функция $b(\alpha)$ строго убьвает на полуинтервале $(1,2]$ и при этом $b(2)=0$, следовательно, величина $b(\alpha)$ отрищательна при всех $\alpha \in(1,2)$. Таким образом, функция $f_{Q}$ имеет экспоненциальное убьвание при $y \rightarrow+\infty$.

Теперь рассмотрим семейство полиномов

$$
\left\{p_{k}(x)=Q_{k}\left(x^{d}\right)=1-\frac{2^{k}}{k^{\alpha}} x^{d}\right\}_{k \in \mathbb{N}}
$$

для некоторого четного $d$ и для некоторого $\alpha \in(1,2)$, выбранного таким образом, что $d / \alpha>d-\varepsilon(\varepsilon>0$ дано). Используя $(9)$, получаем

$$
|f(x)|=\left|f_{Q}\left(x^{d}\right)\right| \leqslant e^{b(\alpha)|x|^{d / \alpha}+\omega\left(|x|^{d / \alpha}\right)}
$$

при всех $x$, достаточно больших по модулю. Так как величина $b(\alpha)$ отрицательна, то $|f(x)| \leqslant C e^{-|x|^{d-\varepsilon}}$ для подходящей константы $C$. 
ПримеР 2. Существует последовательность тригонометрических полиномов $\left\{m_{k}\right\}$ такая, что $\operatorname{deg} m_{k}=2, m_{k}(0)=1$ для каждого $k \geqslant 1$, и соответствующая функция $f(\xi)$ имеет экспоненциальное убьвание при $|\xi| \rightarrow \infty$.

ДокАЗАТЕЛЬСТво. Покажем, что для любого $\beta \in(1 / 2,1)$ последовательность

$$
\left\{m_{k}(\xi)=e^{-i \xi} \frac{\cos \xi-\cos \left(k^{\beta} / 2^{k}\right)}{1-\cos \left(k^{\beta} / 2^{k}\right)}\right\}_{k \in \mathbb{N}}
$$

является искомой. Так как $\left|m_{k}(\xi)\right|=\left|m_{k}(-\xi)\right|$ и, следовательно, $|f(\xi)|=|f(-\xi)|$, то мы можем ограничиться случаем $\xi \geqslant 0$. Далее, поскольку $\cos t=1-t^{2} / 2+h(t)$, где $0 \leqslant h(t) \leqslant t^{4} / 24$, мы раскладываем каждый косинус и после элементарных упрощений получаем

$$
\left|m_{k}\left(2^{-k} \xi\right)\right|=\left|\frac{1-\xi^{2} / k^{2 \beta}-\left(2^{2 k+1} / k^{2 \beta}\right)\left(h\left(k^{\beta} / 2^{k}\right)-h\left(\xi / 2^{k}\right)\right)}{1-\left(2^{2 k+1} / k^{2 \beta}\right) h\left(k^{\beta} / 2^{k}\right)}\right| .
$$

Разобьем произведение $f(\xi)$ на три части следуюшим образом:

$$
f(\xi)=\prod_{k \leqslant 6 \log _{2} \xi} m_{k}\left(2^{-k} \xi\right) \prod_{k \in I(y), k>6 \log _{2} \xi} m_{k}\left(2^{-k} \xi\right) \prod_{k \in J(y), k>6 \log _{2} \xi} m_{k}\left(2^{-k} \xi\right),
$$

где $y=\xi^{1 / \beta}$, а числовые множества $I_{y}, J_{y}$ те же самые, что в примере 1 . Оценим каждое из трех произведений.

a) Множители с номерами $k \leqslant 6 \log _{2} \xi$. Так как

$$
\left\|m_{k}\right\|=2 /\left(1-\cos \frac{k^{\beta}}{2^{k}}\right)=2 /\left(\frac{1}{2} \frac{k^{2 \beta}}{2^{2 k}}-h\left(k^{\beta} / 2^{k}\right)\right) \leqslant 2 /\left(\frac{1}{2} \frac{k^{2 \beta}}{2^{2 k}}-\frac{1}{24} \frac{k^{4 \beta}}{2^{4 k}}\right),
$$

имеем

$$
\begin{aligned}
\prod_{k \leqslant 6 \log _{2} \xi}\left|m_{k}\left(2^{-k} \xi\right)\right| & \leqslant \prod_{k \leqslant 6 \log _{2} \xi} \frac{1}{\left(k^{2 \beta} / 2^{2 k+2}\right)\left(1-k^{2 \beta} /\left(12 \cdot 2^{2 k}\right)\right)} \leqslant C_{0} \prod_{k \leqslant 6 \log _{2} \xi} \frac{2^{2 k+2}}{k^{2 \beta}} \\
& \leqslant C_{0} \prod_{k \leqslant 6 \log _{2} \xi} 2^{2 k+2} \leqslant C_{0} 2^{36 \log _{2}^{2} \xi+18 \log _{2} \xi}
\end{aligned}
$$

где

$$
C_{0}=\left(\prod_{k \in \mathbb{N}}\left(1-\frac{1}{12} \frac{k^{2}}{2^{2 k}}\right)\right)^{-1}
$$

- абсолютная константа.

b) Множители с номерами $k \in I(y), k>6 \log _{2} \xi$. Для больших $\xi$

$$
\prod_{k \in I(y)}\left|m_{k}\left(2^{-k} \xi\right)\right| \leqslant 1 .
$$

Доказательство оставляем читателю. Идея: при больших $\xi$ каждый множитель этого произведения меньше единищы. 
c) Множители $c$ номерами $k \in J(y), k>6 \log _{2} \xi$. Знаменатели. Обратимся к формуле (10) и оценим произведение знаменателей. Поскольку

$$
\frac{2^{2 k+1}}{k^{2 \beta}} h\left(\frac{k^{\beta}}{2^{k}}\right) \leqslant \frac{2^{2 k+1}}{k^{2 \beta}} \frac{1}{24} \frac{k^{4 \beta}}{2^{4 k}}=\frac{1}{12} \frac{k^{2 \beta}}{2^{2 k}}
$$

мы видим, что произведение

$$
\prod_{k \in \mathbb{N}}\left(1-\frac{2^{2 k+1}}{k^{2 \beta}} h\left(\frac{k^{\beta}}{2^{k}}\right)\right)
$$

сходится к некоторой положительной константе $C_{1}$. Ясно, что

$$
\prod_{k \in J(y), k>6 \log _{2} \xi}\left(1-\frac{2^{2 k+1}}{k^{2 \beta}} h\left(\frac{k^{\beta}}{2^{k}}\right)\right) \geqslant C_{1} .
$$

Числители. Имеем

$$
\begin{aligned}
& \prod_{k \in J(y), k>6 \log _{2} \xi}\left|1-\frac{\xi^{2}}{k^{2 \beta}}-\frac{2^{2 k+1}}{k^{2 \beta}}\left(h\left(\frac{k^{\beta}}{2^{k}}\right)-h\left(\frac{\xi}{2^{k}}\right)\right)\right| \\
= & \prod_{k \in J(y), k>6 \log _{2} \xi}\left|1-\frac{\xi^{2}}{k^{2 \beta}}\right|\left|1-\frac{\left(2^{2 k+1} / k^{2 \beta}\right)\left[h\left(k^{\beta} / 2^{k}\right)-h\left(\xi / 2^{k}\right)\right]}{1-\xi^{2} / k^{2 \beta}}\right| .
\end{aligned}
$$

Если $\xi$ достаточно велико, то для всех $k \in J(y)$

$$
\left|1-\frac{\xi^{2}}{k^{2 \beta}}\right|=\left|1-\left(\frac{y}{k}\right)^{2 \beta}\right| \geqslant\left|1-\left(\frac{y}{y+2}\right)^{2 \beta}\right| \geqslant \frac{2}{y+2} \geqslant \frac{1}{y}=\xi^{-1 / \beta} \geqslant \xi^{-2} .
$$

Тогда

$$
\begin{aligned}
& \quad \prod_{k \in J(y), k>6 \log _{2} \xi}\left|1-\frac{2^{2 k} /\left(k^{2 \beta}\right)\left[h\left(k^{\beta} / 2^{k}\right)-h\left(\xi / 2^{k}\right)\right]}{1-\xi^{2} / k^{2 \beta}}\right| \\
& \leqslant \prod_{k \in J(y), k>6 \log _{2} \xi}\left(1+\frac{2^{2 k} /\left(k^{2 \beta} \cdot 24\right)\left(k^{4 \beta} / 2^{4 k}+\xi^{4} / 2^{4 k}\right)}{\xi^{-2}}\right) \\
& \leqslant \prod_{k \in J(y), k>6 \log _{2} \xi}\left(1+\left(\frac{\xi^{6}}{2^{k}}\right)^{1 / 3} \frac{k^{2 \beta}}{24 \cdot 2^{5 k / 3}}+\frac{\xi^{6}}{2^{k}} \frac{1}{24 \cdot 2^{k} k^{2 \beta}}\right) \\
& \leqslant \prod_{k \in J(y), k>6 \log _{2} \xi}\left(1+\frac{k^{2 \beta}}{24 \cdot 2^{5 k / 3}}+\frac{1}{24 \cdot 2^{k} k^{2 \beta}}\right) \leqslant C_{2} .
\end{aligned}
$$

Теперь воспользуемся результатом примера 1 для случая $d=2$ и $\alpha=2 \beta$. Имеем

$$
\prod_{k \in J(y)}\left|1-\frac{\xi^{2}}{k^{2 \beta}}\right| \leqslant e^{b(2 \beta) \xi^{1 / \beta}+\omega\left(\xi^{1 / \beta}\right)} .
$$


Кроме того, для достаточно больших $\xi$

$$
\prod_{k \leqslant 6 \log _{2} \xi}\left|1-\frac{\xi^{2}}{k^{2 \beta}}\right| \geqslant\left(\frac{\xi^{2}}{36 \log _{2}^{2} \xi}-1\right)^{6 \log _{2} \xi} .
$$

Совместив полученные неравенства, вьводим окончательно

$$
\left|\prod_{k \in J(y), k>6 \log _{2} \xi} m_{k}\left(2^{-k} \xi\right)\right| \leqslant \frac{C_{2}}{C_{1}} e^{b(2 \beta) \xi^{1 / \beta}+\omega\left(\xi^{1 / \beta}\right)}\left(\frac{\xi^{2}}{36 \log _{2}^{2} \xi}-1\right)^{-6 \log _{2} \xi} .
$$

Осталось подставить неравенства a), b) и с) в (11) и получить

$$
\begin{aligned}
|f(\xi)| & \leqslant C_{0} 2^{36 \log _{2}^{2} \xi+18 \log _{2} \xi} \cdot \frac{C_{2}}{C_{1}} e^{b(2 \beta) \xi^{1 / \beta}+\omega\left(\xi^{1 / \beta}\right)}\left(\frac{\xi^{2}}{36 \log _{2}^{2} \xi}-1\right)^{-6 \log _{2} \xi} \\
& \leqslant e^{b(2 \beta) \xi^{1 / \beta}+\omega_{1}\left(\xi^{1 / \beta}\right)}
\end{aligned}
$$

где $\omega_{1}(y)=o(y)$ при $y \rightarrow+\infty$. Теперь при любом значении $\varepsilon>0$ мы можем выбрать такое $\beta \in(1 / 2,1)$, что $f(\xi)=O\left(e^{-|\xi|^{2-\varepsilon}}\right)$.

4. Критерий быстрейшего убывания. В этом пункте мы собираемся сформулировать условия, при которых функция $f$ имеет максимально возможную скорость убывания (см. замечание 3). Мы будем говорить, что последовательность тригонометрических полиномов $\left\{m_{k}\right\}_{k \in \mathbb{N}}$ удовлетворяет условию $(*)$, если существует положительная константа $C_{0}$ такая, что для всех достаточно больших $k \operatorname{deg} m_{k}=n$ и все $n$ корней полинома $\widetilde{m}_{k}$ лежат в круге $|z+1| \leqslant C_{0} 2^{-k}$. Оказьвается, что это условие эквивалентно максимальному убьванию.

ТЕорема 2. При условиях теоремы $1 f(\xi)=O\left(\xi^{-n}\right)$ в том и только том случае, если последовательность $\left\{m_{k}\right\}$ удовлетворяет условию $(*)$.

ЗАмЕЧАНИЕ 6 . Согласно данной теореме функция

$$
f(\xi)=\prod m_{k}\left(2^{-k} \xi\right)
$$

имеет максимально возможное убывание, если все корни алгебраических полиномов $\widetilde{m}_{k}$ стремятся к точке -1 достаточно быстро. Легко видеть, что из совокупности условия $(*)$ и условия $m_{k}(0)=1, k \in \mathbb{N}$, следует, что $\left\|m_{k}-w_{n-1}\right\|=O\left(2^{-k}\right)$. Обратное, вообще говоря, неверно. Максимальное расстояние между корнем полинома $\widetilde{m}_{k}$ и точкой -1 может убывать медленнее чем $\left\|m_{k}-w_{n-1}\right\|$.

ДоКАЗАТЕЛЬСТво ТЕОРЕмЫ 2. Необходимость. Поступим так же, как в доказательстве теоремы 1 . Возьмем произвольное $\delta \in(0, \sigma)$ и рассмотрим величину $f\left(2 \pi \cdot 2^{l}+\delta\right)$. По предположению $f\left(2 \pi \cdot 2^{l}+\delta\right)=O\left(2^{-l n}\right)$ при $l \rightarrow \infty$. Применив $(5)$, получаем

$$
\begin{gathered}
\left|m_{l+1}\left(\pi+2^{-l-1} \delta\right) m_{l+2}\left(\frac{\pi}{2}+2^{-l-2} \delta\right) \cdots m_{l+p}\left(2^{1-p} \pi+2^{-l-p} \delta\right)\right|=O\left(2^{-l n}\right) \\
\text { при } l \rightarrow \infty .
\end{gathered}
$$


Согласно теореме $1 m_{j} \rightarrow w_{n-1}$ при $j \rightarrow \infty$, следовательно, $\operatorname{deg} m_{j}=n$ для больших значений $j$ и, более того, для $k$-го множителя $(k=2, \ldots, p)$ в (12) имеем

$$
\lim _{l \rightarrow \infty} m_{l+k}\left(2^{1-k} \pi+2^{-l-k} \delta\right)=w_{n-1}\left(2^{1-k} \pi\right) \neq 0
$$

Таким образом, из (12) следует, что $\left|m_{l+1}\left(\pi+2^{-l-1} \delta\right)\right|=O\left(2^{-l n}\right)$ при $l \rightarrow \infty$. Обозначим через $\left\{z_{s}\right\}_{s=1}^{n}$ корни полиномов $\widetilde{m}_{l+1}$; таким образом,

$$
\widetilde{m}_{l+1}(z)=a_{l+1} \prod_{s=1}^{n}\left(z-z_{s}\right)
$$

где по теореме $1 \lim _{l \rightarrow \infty} a_{l+1}=2^{-n} \quad\left(2^{-n}-\right.$ старший коэффициент полинома $\left.w_{n-1}\right)$. Положим теперь $\rho=\max _{s}\left|z_{s}+1\right|$; без ограничения общности полагаем $\left|z_{n}+1\right|=\rho$. Так же, как в доказательстве теоремы 1 , окружая каждую точку $z_{1}, \ldots, z_{n-1}$ кругом радиуса $r=\sigma /\left(2^{l+1} \pi(n-1)\right)$, показьваем, что существует $\delta_{0} \in(0, \sigma)$, для которого

$$
\prod_{s=1}^{n-1}\left|e^{-i\left(\pi+2^{-l-1} \delta_{0}\right)}-z_{s}\right| \geqslant r^{n-1} .
$$

Следовательно, $\left|m_{l+1}\left(\pi+2^{-l-1} \delta_{0}\right)\right| \geqslant\left|a_{l+1}(\rho-r) r^{n-1}\right|$, поэтому

$$
a_{l+1}(\rho-r) 2^{-(l+1)(n-1)}=O\left(2^{-l n}\right),
$$

из чего заключаем $\rho=O\left(2^{-l}\right)$ при $l \rightarrow \infty$.

Достаточность. Мы проведем доказательство для положительных $\xi$ (случай отрицательных $\xi$ рассматривается аналогично). Далее, мы можем ограничиться рассмотрением больших значений $\xi$, поэтому полагаем $\xi \geqslant 4 \pi$. Более того, достаточно провести доказательство лишь для случая $n=1$. Иначе говоря, мы докажем следующее: если последовательность комплексных чисел $\left\{z_{k}\right\}$ удовлетворяет условию $(*)$, т.е. сходится к точке -1 так, что $\left|z_{k}+1\right| \leqslant C_{0} 2^{-k}$, то

$$
\left|f_{1}(\xi)\right|=\left|\prod_{k=1}^{\infty} \frac{e^{-i 2^{-k} \xi}-z_{k}}{2}\right|=O\left(\frac{1}{\xi}\right) \quad \text { при } \quad \xi \rightarrow+\infty .
$$

Действительно, если мы разложим каждый полином

$$
m_{k}(\xi)=a_{k} \cdot 2^{n}\left(\frac{e^{-i \xi}-z_{1 k}}{2}\right) \cdots\left(\frac{e^{-i \xi}-z_{n k}}{2}\right)
$$

и заметим, что в предположениях теоремы $2\left|a_{k} \cdot 2^{n}-1\right|=O\left(2^{-k}\right)$ при $k \rightarrow \infty$ (замечание 6$)$, то получим, что последовательность полиномов $\prod_{k \in \mathbb{N}}\left(a_{k} \cdot 2^{n}\right)$ сходится к некоторой константе. Теперь, применив (13) к каждой последовательности полиномов

$$
\left\{\left(\frac{e^{-i \xi}-z_{s k}}{2}\right)\right\}_{k \in \mathbb{N}}, \quad s=1, \ldots, n,
$$


завершаем доказательство теоремы. Осталось доказать (13).

Обозначим $\xi=2 \pi x$ и представим $x$ в двоичном разложении: $x=d_{1} \ldots d_{l} . d_{l+1} \ldots$ Рассмотрим последовательность цифр перед точкой: $d_{1} \ldots d_{l}$. Она начинается с нескольких последовательных единиц $d_{1} \ldots d_{k_{1}}$, затем следует последовательность нулей $d_{k_{1}+1} \ldots d_{k_{2}}$ и т.д. Предположим, что последняя цифра $d_{l}$ - нуль (случай единицы рассматривается аналогично), таким образом, последовательность оканчивается серией нулей $d_{k_{s}+1} \ldots d_{l}$. Наконец, положим $D=\left\{k_{1}, \ldots, k_{s}\right\}$ - последовательность последних цифр серий. Обозначим $h_{k}(\xi)=\left(e^{-i 2^{-k} \xi}-z_{k}\right) / 2$ и разложим произведение (13) на три части:

$$
f_{1}(2 \pi x)=\left(\prod_{k=1}^{l-k_{s}} h_{k}\right) \cdot h_{l-k_{s}+1} \prod_{k=l-k_{s}+2}^{\infty} h_{k} .
$$

Оценим каждую из частей по отдельности.

а) Поскольку

$$
\left|h_{k}\right| \leqslant \frac{1+\left|z_{k}\right|}{2} \leqslant \frac{2+\left|z_{k}-1\right|}{2} \leqslant 1+C_{0} 2^{-k-1} \leqslant e^{C_{0} 2^{-k-1}}
$$

получаем

$$
\left|\prod_{k=1}^{l-k_{s}} h_{k}(2 \pi x)\right| \leqslant e^{C_{0} / 2} .
$$

b) Имеем $h_{l-k_{s}+1}(2 \pi x)=\left(e^{-2 \pi i \bar{x}}-z_{l-k_{s}+1}\right) / 2$, где

$$
\bar{x}=\left\{2^{k_{s}-l-1} x\right\}=2^{k_{s}-l-1} x-\left[2^{k_{s}-l-1} x\right]=0.10 \ldots 0 d_{l+1} \ldots
$$

$\left(l-k_{s}\right.$ нулей после точки). Итак, $|2 \pi \bar{x}-\pi| \leqslant \pi 2^{k_{s}-l}$, поэтому

$$
\begin{aligned}
\left|\frac{e^{-2 \pi i \bar{x}}-z_{l-k_{s}+1}}{2}\right| & \leqslant \frac{\left|e^{-2 \pi i \bar{x}}-e^{-i \pi}\right|+\left|e^{-i \pi}-z_{l-k_{s}+1}\right|}{2} \leqslant \frac{\pi 2^{k_{s}-l}+C_{0} 2^{k_{s}-l-1}}{2} \\
& \leqslant\left(2 \pi+C_{0}\right) 2^{k_{s}-l-2} .
\end{aligned}
$$

c) Имеем

$$
\begin{aligned}
\prod_{k=l-k_{s}+2}^{\infty}\left|h_{k}\right| & =\left|\prod_{k=l-k_{s}+2}^{\infty} \frac{e^{-2 \pi i 2^{-k} x}+1}{2}\right| \cdot\left|\prod_{k=l-k_{s}+2}^{\infty}\left(1-\frac{z_{k}+1}{e^{-2 \pi i 2^{-k} x}+1}\right)\right| \\
& \leqslant\left|\prod_{k=l-k_{s}+2}^{\infty} w_{0}\left(2^{-k} \cdot 2 \pi x\right)\right| \cdot \prod_{k=l-k_{s}+2}^{\infty}\left(1+\left|\frac{z_{k}+1}{e^{-2 \pi i 2^{-k} x}+1}\right|\right) \\
& \leqslant \frac{2}{2^{k_{s}-l-1} \cdot 2 \pi x} \prod_{k=l-k_{s}+2}^{\infty}\left(1+\left|\frac{z_{k}+1}{e^{-2 \pi i 2^{-k} x}+1}\right|\right) \\
& \leqslant \frac{2}{2^{k_{s}-l-1} \cdot 2 \pi x} \exp \left(\sum_{k \geqslant l-k_{s}+2}\left|\frac{z_{k}+1}{e^{-2 \pi i 2^{-k} x}+1}\right|\right)
\end{aligned}
$$


Для того чтобы оценить слагаемые в последней сумме, рассмотрим два возможных случая.

1) В двоичной записи числа $\bar{x}=\left\{2^{-k} x\right\}$ две первые цифры после точки равны. Это означает, что число $k$ не представляется в форме $k=l-k_{j}+1$ ни для какого $k_{j} \in D$, иными словами, $l-k+1 \notin D$. Тогда $|\bar{x}-1 / 2| \geqslant 1 / 4$, следовательно, $\left|e^{-2 \pi i \bar{x}}+1\right|>1$ и, таким образом,

$$
\left|\frac{z_{k}+1}{e^{-2 \pi i 2^{-k} x}+1}\right| \leqslant\left|z_{k}+1\right| \leqslant C_{0} 2^{-k}
$$

Итак,

$$
\sum_{\substack{k \geqslant l-k_{s}+2 \\ l-k+1 \notin D}}\left|\frac{z_{k}+1}{e^{-2 \pi i 2^{-k} x}+1}\right| \leqslant \sum_{\substack{k \geqslant l-k_{s}+2 \\ l-k+1 \notin D}} C_{0} 2^{-k} \leqslant \sum_{k \in \mathbb{N}} C_{0} 2^{-k} \leqslant C_{0} .
$$

2) В двоичной записи числа $\bar{x}$ две первые цифры после точки различны, т.е. число $k$ представляется в форме $k=l-k_{j}+1$ для некоторого $k_{j} \in D$. В этом случае $|\bar{x}-1 / 2| \geqslant 2^{k_{j}-k_{j+1}-1}$, поэтому $\left|e^{-2 \pi i \bar{x}}+1\right| \geqslant 2^{k_{j}-k_{j+1}+1}$. Тогда

$$
\begin{aligned}
\sum_{\substack{k \geqslant l-k_{s}+2 \\
l-k+1 \in D}}\left|\frac{z_{k}+1}{e^{-2 \pi i 2^{-k} x}+1}\right| \leqslant \sum_{\substack{k=l-k_{j}+1 \\
k_{j} \in D}} \frac{C_{0} 2^{-k}}{2^{k_{j}-k_{j+1}+1}}=\sum_{j=1}^{s} \frac{C_{0} 2^{k_{j}-l-1}}{2^{k_{j}-k_{j+1}+1}} \\
=\sum_{j=1}^{s} C_{0} 2^{k_{j+1}-l-2} \leqslant \sum_{k=1}^{l} C_{0} 2^{k-l-2}<\frac{1}{2} C_{0} .
\end{aligned}
$$

Применив теперь (14), получаем

$$
\sum_{k \geqslant l-k_{s}+2}\left|\frac{z_{k}+1}{e^{-2 \pi i 2^{-k} x}+1}\right| \leqslant \frac{3}{2} C_{0}
$$

Итак, в случае с) имеем

$$
\prod_{k \geqslant l-k_{s}+2}\left|h_{k}\right| \leqslant \frac{1}{2^{k_{s}-l-2} \cdot 2 \pi x} e^{3 C_{0} / 2}
$$

Теперь случаи а), b) и с) вместе дают

$$
\begin{aligned}
\left|f_{1}(\xi)\right| & =\left|f_{1}(2 \pi x)\right|<e^{C_{0} / 2} \cdot\left(2 \pi+C_{0}\right) 2^{k_{s}-l-2} \cdot \frac{1}{2 \pi \cdot 2^{k_{s}-l-2} x} e^{3 C_{0} / 2} \\
& \leqslant \frac{e^{2 C_{0}}\left(2 \pi+C_{0}\right)}{2 \pi x}=\frac{e^{2 C_{0}}\left(2 \pi+C_{0}\right)}{\xi},
\end{aligned}
$$

что завершает доказательство.

Автор выражает признательность профессору Воронежского государственного университета И.Я. Новикову и профессору Московского государственного университета С. В. Конягину за полезные обсуждения и постоянное внимание к данной работе. 


\section{СПИСОК ЦИТИРОВАННОЙ ЛИТЕРАТУРЫ}

[1] Derfel G. A. A probabilistic method for a class of functional-differential equations // Ukrain. Math. J. 1989. V. 41. №10. P. 1137-1141.

[2] Derfel G. A., Dyn N., Levin D. Generalized refinement equations and subdivision processes // J. Approximation Theory. 1995. V. 80. P. 272-297.

[3] Protasov V. Refinement equations with nonnegative coefficients // J. Fourier Anal. Appl. 2000. V. 6. №6. P. 55-78.

[4] Cavaretta D., Dahmen W., Micchelli C. Stationary subdivision // Mem. Amer. Math. Soc. 1991. V. 93. P. 1-186.

[5] Dyn N.,Gregory J. A., Levin D. Analysis of linear binary subdivision schemes for curve design // Constructive Approximation. 1991. V. 7. P. 127-147.

[6] Erdös P. On the smoothness properties of Bernoulli convolutions // Amer. J. Math. 1940. V. 62. P. $180-186$.

[7] Peres Y., Solomyak B. Absolute continuity of Bernoulli convolution, a simple proof // Math. Res. Letters. 1996. V. 3. № 2. P. 231-239.

[8] Reznick B. Some binary partition functions // Analytic Number Theory (Allerton Park II, 1989). P. 451-477.

[9] Chui C. K. An Introduction to Wavelets // Acad. Press Inc. 1992.

[10] Dyn N., Levin D. Interpolatory subdivision schemes for the generation of curves and surfaces // Multivariate Approximation and Interpolation (Duisburg, 1989). Basel: Birkhäuser, 1990. P. 91-106.

[11] Ron A. Smooth refinable functions provide good approximation // SIAM J. Math. Anal. Appl. 1997. V. 28. P. 731-748.

[12] Daubechies I. Orthonormal bases of wavelets with compact support // Comm. Pure Appl. Math. 1988. V. 41. P. 909-996.

[13] Cohen A., Daubechies I. A new technique to estimate the regularity of refinable functions // Rev. Mat. Iberoamericana. 1996. V. 12. № 2. P. 527-591.

[14] Ron A., Shen Z. The Sobolev Regularity of Refinable Functions. Preprint, 1997.

[15] Daubechies I., Lagarias J. Two-scale difference equations. I. Global regularity of solutions // SIAM J. Math. Anal. Appl. 1991. V. 22. P. 1388-1410.

[16] Daubechies I., Lagarias J. Two-scale difference equations. II. Local regularity, infinite products of matrices and fractals // SIAM J. Math. Anal. Appl. 1992. V. 23. P. 1031-1079.

[17] Schumaker L. L. Spline Functions: Basic Theory. New York: John Wiley, 1981.

[18] Берколайко М.З., Новиков, И. Я. О бесконечно гладких почти-всплесках с компактным носителем // Докл. РАН. 1992. Т. 326. №6. С. 615-618.

[19] de Boor C., DeVore R., Ron A. On the construction of multivariate pre-wavelets // Constructive Approximation. 1993. V. 2. № 3. P. 123-166.

[20] Новиков И. Я. Базисы всплесков функциональных пространств. Дисс. . . докт. физ.-матем. наук. Воронеж: Воронежский государственный университет, 2000.

[21] Cohen A., Dyn N. Nonstationary subdivision schemes and multiresolution analysis // SIAM J. Math. Anal. Appl. 1996. V. 27. P. 1745-1769. 